\title{
Design of current sources for load common mode optimization
}

\author{
Vinicius G. Sirtoli ${ }^{1,2}$, Kaue F. Morcelles ${ }^{1}$ and Volney C. Vincence ${ }^{1}$ \\ 1. Department of Electrical Engineering, Santa Catarina State University (UDESC), Joinville, Brazil \\ 2. E-mail any correspondence to: vinicius.sirtoli@gmail.com
}

\begin{abstract}
Bioimpedance measurement systems often use the Howland current sources to excite the biological material under study. Usually, difference or instrumentation amplifiers are used to measure the resulting voltage drop on this material. In these circuits, common mode voltage appears as artifacts in the measurement. Most researches on current sources are focused on improving the output impedance, letting other characteristics aside. In this paper, it is made a brief review on the load common mode voltage and output swing of various topologies of Howland current sources. Three circuits are proposed to reduce load common mode voltage and enhance load capability by using a fully differential amplifier as active component. These circuits are equated, simulated and implemented. The three proposed circuits were able to deliver an output current with cut-off frequency (-3dB) higher than $1 \mathrm{MHz}$ for loads as big as $4.7 \mathrm{k} \Omega$. The worst measured load common mode voltage was smaller than $24 \mathrm{mV}$ for one of the circuits and smaller than $8 \mathrm{mV}$ for the other two. Consequently, it could be obtained increases in the Common Mode Rejection Ratio (CMRR) up to $60 \mathrm{~dB}$ when compared to the Enhanced Howland Current Source (EHCS).
\end{abstract}

Keywords: Bioimpedance, Howland current source, Common mode rejection

\section{Introduction}

Electrical Bioimpedance Spectroscopy (EBIS) is a technique to assess passive electrical properties of a biological material [1]. It can be invasive, non-invasive or a combination of both. To measure the bioimpedance, it is injected an electrical signal and acquired the biological material electrical response. There are many applications of EBIS, and a lot of them involve the diagnosis of diseases such as skin cancer [2], pressure ulcer [3] and brain cellular edema [4]. Highly precise instruments for both signal generation and acquisitions are essential to have accurate diagnosis.

In practice, many EBIS systems use a current source to excite the biological material instead of a voltage source. In this case, the voltage across the load is measured and the impedance is calculated. This means that the higher the injected current, the higher the measured voltage, and consequently, the higher the signal to noise ratio. Therefore, it is essential to have a current source that can deliver a fixed amplitude current over all frequency range, assuring maximum resolution [5]. Moreover, the output current amplitude can easily be designed to meet medical safety that is usually adopted by researchers as $1 \mathrm{~mA}$.

On the other hand, practical current sources have shown worse high frequency performance in comparison to voltage excitation systems [6-8]. This can be explained by the decrease in the open loop gain of the amplifier and stray capacitances at the circuit, which degrades the overall output impedance as frequency increases [9]. Researchers are trying to create or modify circuits to develop a current source that can work in a wider frequency range.

In bioimpedance applications most systems use a voltage controlled current source (VCCS), usually the enhanced Howland current source (EHCS), because it has shown to be very simple and robust [10]. A few modifications have been proposed to this circuit such as: Mirrored [11] and Bridge [12] configurations, Load-in-theloop [13], NIC [14] and GIC [15] output stages, high frequency compensations such as Lead-Lag circuits [10], and by using differential amplifiers as the active 
components [16]. However, most of these improvements focus in the output impedance and bandwidth flatness, leaving characteristics like swing and load common mode voltage aside.

The total impedance seen by the current source is two times the electrode impedance plus the tissue impedance, which is unknown. Thus, higher load capability leads to broader range of applications. Further, if a common mode voltage is generated across the load, artifacts can be generated in the measurement, leading to errors [17], especially in high frequency, where the CMRR of the instrumentation amplifiers decay significantly.

Another commonly ignored issue is the electrode polarization that happens mainly in inert metallic materials (stainless steel, gold and platinum) but also in graphite and a few organic materials $[16,18]$. The electrode polarization is a result of the double layer developed at the electrodeelectrolyte interface.

In order to solve or attenuate some of the problems mentioned above researchers used symmetrical output current sources. Pliquett et al. developed a variation of the Enhanced Howland Current Source [EHCS] based on fullydifferential amplifiers to be used with microelectrodes [16]. Liu et al. developed a Howland Current Source with an auxiliary circuit that copies the voltage in one side of the load to the other one, the application intended was Electrical Impedance Tomography [EIT] [17]. Recently, Zhang et al. developed a Mirrored Current Source using the IC AD844 (current-conveyor) to reduce analog common mode signals and digital common mode noise [19]. Due to low voltage power supply and relatively high current along with high interface electrode-interface impedance caused by effects like fibrosis, Lamlih et al. developed an integrated differential current source to maximize the voltage output swing [20].

Swing and load common mode voltage of Howland current sources are analyzed in this work. Comparisons between different topologies are made in simulations. Finally, three new circuits are proposed based on differential amplifiers. Equations, simulations and implementation results are presented to validate the new topologies.

\section{Materials and methods}

The Howland current sources are split in three groups: nondifferential, mirrored and proposed. The Theory Section presents the analytical study of load common mode voltage for each topology.

The three proposed circuits are explained and then modelled. All the equations were obtained using wxMaxima. The equations define the output current, the output impedance and the maximum load as function of the amplifier output saturation voltage.
The circuits are simulated with Orcad Pspice, the simulations are done in the frequency domain (Bode plot) to observe the $A C$ response, and in the time domain to observe the waveform. The AD8132 spice model was obtained from the manufacturer web page and was used in all simulations. The simulated data is plotted and compared to the equations in MATLAB.

All proposed circuits are implemented in a two layer PCB, the operational amplifier used is the AD8132 and all resistors have $1 \%$ of tolerance (trough-hole components). The common mode input pin of the AD8132 can be used to reduce the common mode voltage between the outputs, but in this work, it was grounded so it would not influence the circuit performance, hence the proposed circuits can be reproduced with other differential amplifiers that do not have this pin. Furthermore, Pliquett et al. also grounded this pin [18].

The signal is generated with a Tektronix function generator (model AFG 3251) and measured with a Tektronix oscilloscope (model MSO 4034).

The input frequency is varied from $100 \mathrm{~Hz}$ to $1 \mathrm{MHz}$. The loads used are four resistors of $100 \Omega, 995 \Omega, 2492 \Omega$ and $4692 \Omega$. Although the bioimpedance may vary from a few $\Omega$ to hundreds of $k \Omega$ depending on the material under test and the electrode used, it is believed that the load range used is enough to demonstrate the objective of this work, whereas other works have used close values $[9,13$, $19,21,22]$.

The data of the two voltages across the load and the input voltage are saved from the oscilloscope and exported to MATLAB, where the Fast Fourier Transform (FFT) algorithm is applied to the signals and the output current magnitude, phase and load common mode voltage are calculated.

\section{Ethical approval}

The conducted research is not related to either human or animal use.

\section{Theory}

The enhanced Howland current source (EHCS), shown in Fig. 1, is a voltage controlled current source (VCCS), where the output current (lout), as a function of the input voltage $\left(V_{i n}\right)$, is given by the equation 1 .

$$
I_{\text {out }}=\frac{V_{\text {in }}}{r}
$$

Many modifications have been proposed to this circuit to enhance some of its characteristics, such as output current bandwidth, output impedance, output swing and common mode current.

The voltage across the load has a differential and common mode component. The differential one affects the output swing capability, while the common mode affects the quality of the measurement. 
The common mode rejection ratio (CMRR) is the relation between the differential and the common-mode parts of the signal, usually expressed in $\mathrm{dB}$.

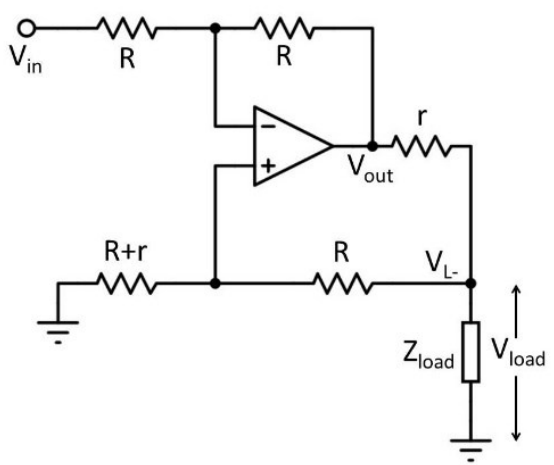

Fig.1 Enhanced Howland Current Source (EHCS).

To investigate the load voltage on different topologies of Howland current sources, they were split into 3 groups: non-differential, mirrored, and proposed. Each one will be discussed individually in the following subsections.

\section{Non-differential Group}

The non-differential group includes the Howland current sources that have only one current output. One side is called the current side and the other voltage side. It can be single (EHCS), where the voltage side is connected to the ground, or floating (EHCS-BRIDGE1, EHCS-BRIDGE2, EHCSDIFFERENTIAL).

The EHCS, shown in Fig. 1, has one side of the load grounded, thus completely concentrating load voltage on the node $V_{L-}$ (see Fig. 1). This requires that the entire voltage drop on the load be provided by the current source, limited by the EHCS compliance.

If the other node of the load is connected to an

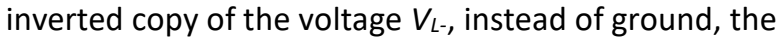
EHCS will need to yield only half of the load voltage drop (see Eq. 2 and 3), thus improving the overall compliance. This result can be achieved by a differential approach.

$$
\begin{gathered}
V_{\text {load }}=V_{L_{-}}-\left(-V_{L-}\right)=2 V_{L-} \\
V_{L-}=\frac{V_{\text {load }}}{2}
\end{gathered}
$$

Equation 4 describes the common mode signal in function of the voltage on the negative $\left(V_{L-}\right)$ and positive $\left(V_{L^{+}}\right)$nodes of the load on an arbitrary current source. Common mode voltage is significant when using the EHCS, because the node $V_{L+}$ is grounded, which results in a $V_{L c m}$ equal to half of the voltage drop across the load, degrading the signal to noise ratio of measurements.

$$
V_{L c m}=\frac{V_{L+}+V_{L-}}{2}
$$

In order to eliminate common mode problems, the circuit must provide a differential output, yielding $V_{L-}=-V_{L+}$.
This approach also doubles the compliance (see Eq. 2), suggesting that a differential approach is better suited to increase common mode signal rejection and load capability. Furthermore, high symmetrical output current sources are partially able to compensate the effects of the double layer [18].

The circuit in Fig. 2, based on [12] and called here EHCSBRIDGE-1, uses a unitary gain inverter to obtain $-V_{\text {out }}$. To achieve differential balance, a resistor $\left(r_{\text {trim }}\right)$ is placed in series with the load.

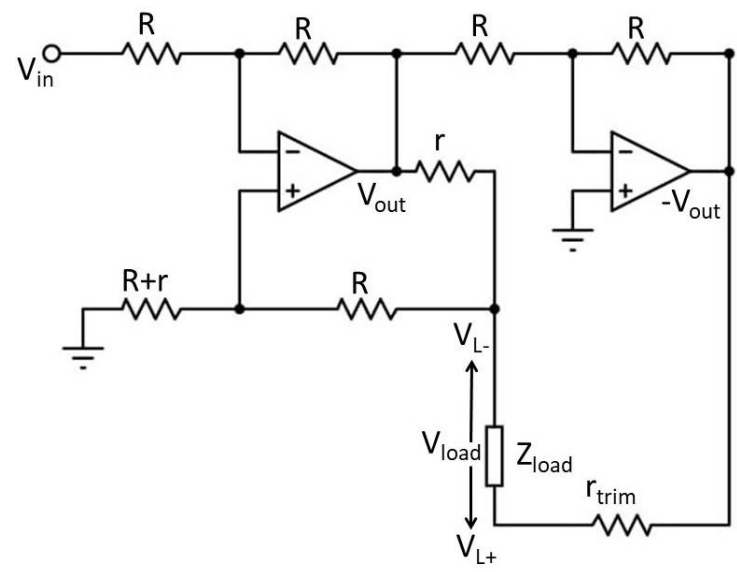

Fig.2: Enhanced Howland Current Source with Bridge topology (BRIDGE-1)

The value of $r_{\text {trim }}$ must be set to mimic the voltage drop

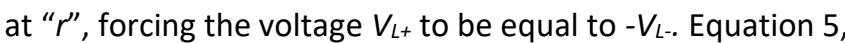
adapted from Tucker [10], models $V_{L}$ - in terms of $V_{\text {out }}$ and $V_{\text {in }}$ (see Fig. 1) considering the positive feedback current passing through $\mathrm{R}$ much lower than the output one. It can be seen that the voltage drop across the resistor " $r$ " is approximately $V_{i n}$, so the value of $r_{\text {trim }}$ can be calculated once the output current is known.

$$
V_{L_{-}} \approx V_{\text {out }}-V_{\text {in }}
$$

The EHCS-BRIDGE-1 can be simplified using a differential output amplifier to generate $-V_{\text {out }}$ instead of an additional inverting amplifier [16], as it can be seen in Fig. 3. The appropriate value of $r_{\text {trim }}$ is the same from EHCSBRIDGE-1.

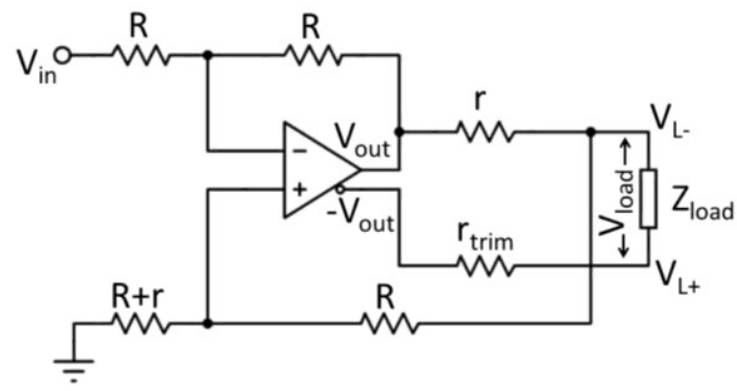

Fig.3: Enhanced Howland Current Source with differential amplifier (EHCS-DIF). 
However, Eq. 5 is only an approximation, as the current passing through $r$ varies with the load value (due to the feedback current increase), changing its voltage drop. Because $r_{\text {trim }}$ remains the same, an unbalance is developed across the load. Thus, the common mode voltage and output swing are dependent on the load value. This shows a disadvantage of the topology, and will be further discussed later.

The unbalance caused by $r_{\text {trim }}$ can be overcome by the topology shown in Fig. 4, based on [17]. The circuit uses a buffer at $V_{L}$ - in series with an inverting amplifier to generate $-V_{L-}$ at the voltage side of the load, so $r_{\text {trim }}$ is not necessary.

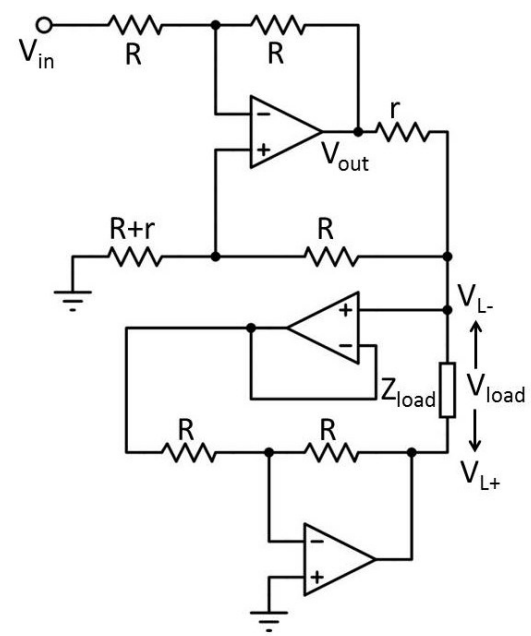

Fig.4: Enhanced Howland Current Source with Bridge topology (BRIDGE-2).

However, an additional amplifier acting as a buffer is needed, to isolate the inverter and avoid loading, which increases complexity, noise and parasitic capacitance at the output.

\section{Mirrored Group}

The mirrored group consists of two circuits: the Mirrored Enhanced Howland Current Source (MEHCS) [11] (Fig. 5) and a proposed modification of this same circuit using differential amplifiers (MEHCS-DIF) (Fig. 6).

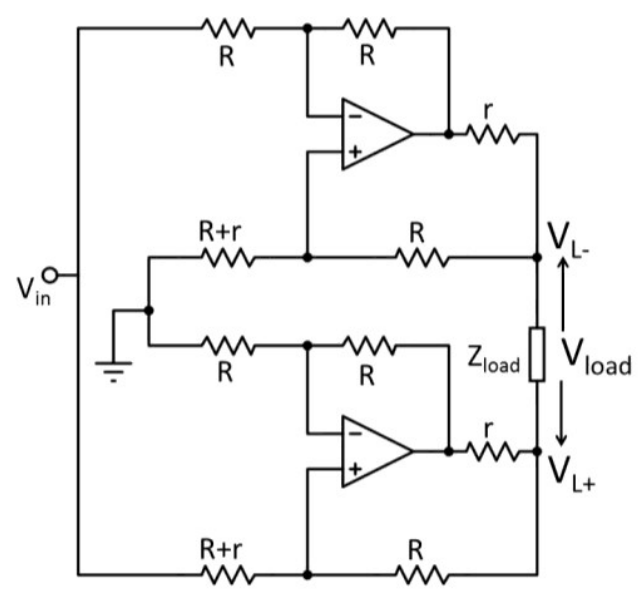

Fig.5: Mirrored Enhanced Howland Current Source (MEHCS).
MEHCS is implemented with two opposite polarity EHCS connected in series. The principle is a symmetrical current generation (source and sink), and consequently inverse voltages at each side of the load.

The problem with the MEHCS is that the output current of each output of this current source is not exactly the same because of circuit mismatches and different input impedances [11]. The output current difference between the source and sink sees the output impedance of the current sources, creating high common mode voltage and reducing output swing.

The use of fully differential amplifiers allows the input voltage to be connected to the same input at both sides, minimizing the difference between input impedances. However, the possibility of circuit mismatches due to component tolerances and temperature drift remains.

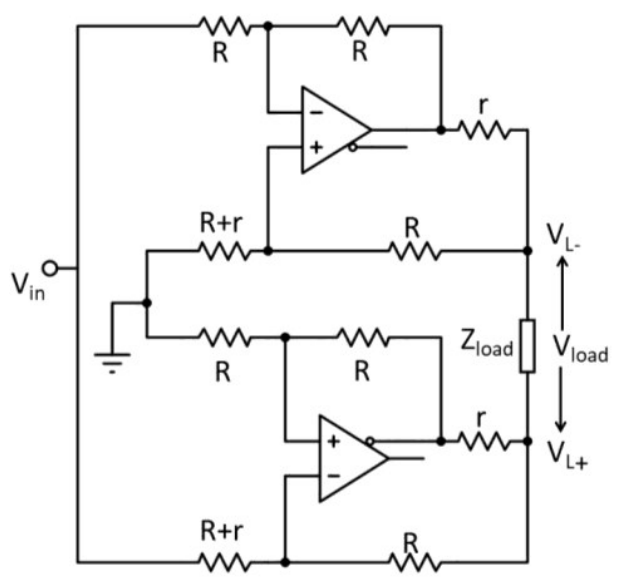

Fig.6: Mirrored Enhanced Howland Current Source with differential amplifiers (MEHCS- DIF).

It can be seen from the previously analysis that most solutions proposed in the literature to improve common mode and output swing needs multiple active devices and matched resistor networks, which results in increase of complexity and, in practice, unbalance problems.

\section{Proposed Group}

The proposed group of circuits uses differential amplifiers to create symmetrical current sources using only one active device. Three topologies are proposed: Quad feedback EHCS (QUAD), Crossed EHCS-DIF (DIF_C) and Modified EHCS-DIF (DIF_M). All circuits are inspired by EHCS-DIF and MEHCS.

The Quad-feedback EHCS uses one differential amplifier to create a mirrored EHCS. Comparing to the standard EHCS from Fig. 1 , it can be seen that $R_{6}$ creates a second negative feedback and $R_{3}$ creates a second positive feedback, while $r_{x 2}$ has the same function as $r_{x 1}$ 


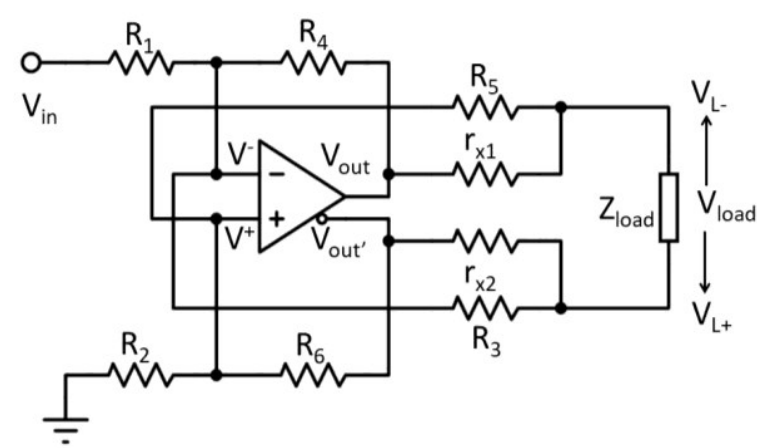

Fig.7: Quad-Feedback Enhanced Howland Current Source with differential amplifiers (QUAD).

To balance the feedbacks, the following considerations were made:

$$
\begin{aligned}
& r_{x 1}=r_{x 2}=r_{x} \\
& R_{1}=R_{2}=R_{c} \\
& R_{4}=R_{6}=R_{b} \\
& R_{3}=R_{5}=R_{a}
\end{aligned}
$$

Considering an ideal case where:

$$
\begin{gathered}
V_{\text {out } \text { I }}=-V_{\text {out }} \\
V^{+}=V^{-}
\end{gathered}
$$

Using the software wxMaxima to rearrange the node equations, it was obtained an equation of $l_{\text {out }}$ as function of $V_{i n}, V_{L-}$ and $V_{L+}$; which is not showed in this work due to its excessive size. In order to simplify the equation, the following consideration was made:

$$
R_{b}=r_{x}+R_{a}
$$

Then, an equation independent from the load is obtained.

$$
I_{\text {out }}=\frac{V_{\text {in }} R_{b}}{2 R_{c} r_{x}}
$$

This equation considers an ideal op-amp, for a more accurate one it can be included the open loop gain $\left(A_{o}\right)$ by changing Eq. 11 for Eq. 14.

$$
V_{\text {out }}=A_{o}\left(V^{+}-V^{-}\right)
$$

Now, the output current equation is given by Eq. 15 .

$$
I_{\text {out }}=\frac{\left[r_{x}^{2}+\left(A_{o}+1\right) R_{a} r_{x}+A_{o} R_{a}^{2}\right] V_{\text {in }}}{\left(R_{c}+R_{a}\right) r_{x}^{2}+\left[\left(2 A_{o}+2\right) R_{a} R_{c}+R_{a}^{2}\right] r_{x}}
$$

The output impedance equation (Eq. 16) is obtained by using the same node equations but grounding the input voltage.

$$
Z_{\text {out }}=\frac{2 r_{x}\left[R_{a}\left(R_{b}+R_{c}\left(2+A_{o}\right)\right)+R_{c} r_{x}\right]}{\left(R_{a}+r_{x}\right)\left(R_{b}+2 R_{c}\right)}
$$

In further analysis, it can be seen that the purpose of the second negative feedback is to create an opposite $V_{\text {out }}$, which is not necessary since it is already done internally by the differential amplifier. Then $\mathrm{R}_{6}$ can be eliminated from the circuit, leading to the EHCS-DIF_C from Fig. 8.

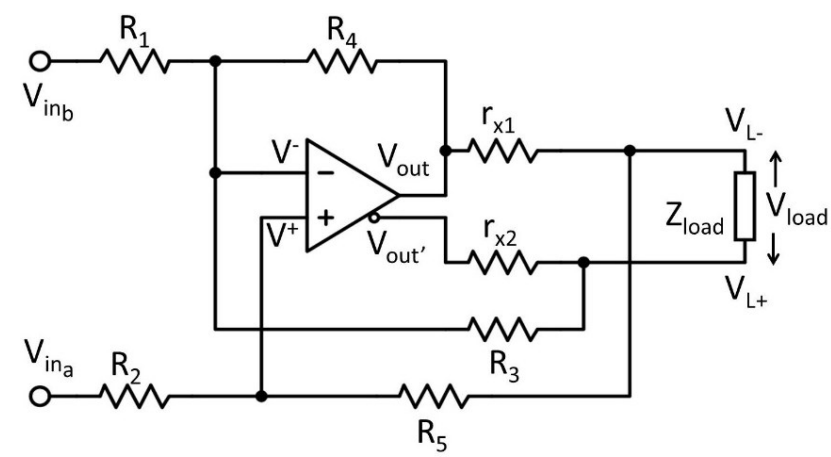

Fig.8: Crossed Differential Enhanced Howland Current Source with differential amplifiers (DIF_C).

To balance the positive feedbacks, their resistors were equaled (Eq.17), while equations 6, 10 and 11 are also valid in this analysis:

$$
R_{3}=R_{5}
$$

Again, using the software wxMaxima, it was obtained an equation of lout as function of $V_{i n}, V_{L}$ and $V_{L+}$; which is not showed in this work due to its excessive size. Eq. 18 and 19 lead to an lout independent from the load (Eq. 20):

$$
\begin{aligned}
& R_{4}=r_{x}+R_{3} \\
& R_{2} \ll r_{x}+R_{3}
\end{aligned}
$$

From Eq. 19 it is assumed that the best case is $R_{2}=0$.

$$
I_{\text {out }}=\frac{\left(r x+R_{3}+2 R_{1}\right) V_{i n_{a}}-\left(r x+R_{3}\right) V_{i n_{b}}}{R_{1} r_{x}}
$$

The inputs $V_{i n_{a}}$ and $V_{i n_{b}}$ sees different transconductances, then the DIF_C topology cannot be used with differential input, as shown in equation 20. However, it is not a problem when one input is used for signal and the other for offset compensation.

Considering the practical case of Eq. 14 instead of the ideal one (Eq. 11), the output current equation can be described as a function of $A_{o}$.

$I_{\text {out }}=\frac{\left(A_{o} R_{3} r x+A_{o} R_{3}^{2}+2 A_{o} R_{1} R_{3}\right) V_{i n_{a}}-\left(r_{x}^{2}+\left(A_{o}+1\right) r_{x} R_{3}+A_{o} R_{3}^{2}\right) V_{i n_{b}}}{\left(R_{3}+R_{1}\right) r_{x}^{2}+\left(R_{3}^{2}+\left(A_{o}+2\right) R_{1} R_{3}\right) r_{x}}$

Using the same node equations and grounding both inputs, it was obtained a simplified equation for the output impedance.

$$
Z_{\text {out }}=\frac{r_{x}^{3}+\left(2 A_{o}+7\right) R_{3} r_{x}^{2}+\left(2 A_{o}+6\right) R_{3}^{2} r_{x}}{3 r_{x}^{2}+6 R_{3} r_{x}+3 R_{3}^{2}}
$$

The third proposed topology is shown in Fig. 9 and is clearly an adaptation of EHCS-DIF, with only the insertion of R5. 


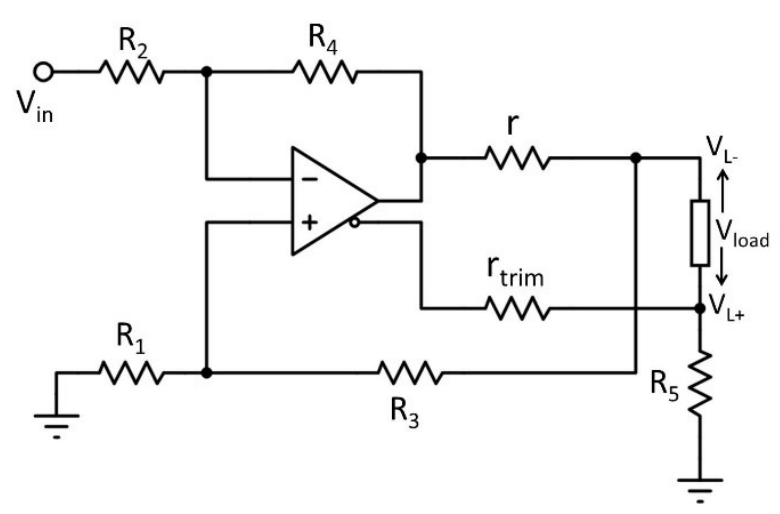

Fig.9: Modified Differential Enhanced Howland Current Source with differential amplifiers (DIF_M).

To make the circuit independent from the load, the same resistors conditions of the EHCS are assumed, this is:

$$
\begin{gathered}
R_{3}=R_{4} \\
R_{2}=R_{4} \\
R_{1}=R_{4}+r
\end{gathered}
$$

Then lout is defined by Eq.1, the same equation for the EHCS output current. If it is considered that the open-loop gain of the op-amp is not infinite then Eq. 26 defines the output current, while the output impedance is described by equation Eq. 27.

$$
\begin{gathered}
I_{\text {out }}=\frac{A_{o} V_{\text {in }}}{\left(A_{o}+2\right) r} \\
Z_{\text {out }}=\frac{r_{x}^{3}+\left(2 A_{o}+7\right) R_{3} r_{x}^{2}+\left(2 A_{o}+6\right) R_{3}^{2} r_{x}}{3 r_{x}^{2}+6 R_{3} r_{x}+3 R_{3}^{2}}
\end{gathered}
$$

It can be seen that the output current follows the same equations as the EHCS. To find out the influence of the load on the value of $r_{\text {trim }}$ and $R_{5}, V_{L c m}$ was modelled in terms of the circuit resistors and then equaled to zero. An equation of $r_{\text {trim }}$ in terms of the others resistors was then obtained (Eq. 28).

$$
r_{\text {trim }}=\frac{2 r^{2}+Z_{\text {load }} r+4 R_{3} r}{\frac{Z_{\text {load }} r}{R_{5}}+2 r+\frac{2 R_{3} Z_{\text {load }}}{R_{5}}+4 R_{3}}
$$

Considering $R_{5}$ infinite, consequently leading back to EHCS-DIF topology, it can be seen the dependence of $r_{\text {trim }}$ on the load on Eq. 29.

$$
r_{\text {trim }}=\frac{\left(2 r+Z_{\text {load }}+4 R_{3}\right) r}{2 r+4 R_{3}}
$$

If $Z_{\text {load }}$ is equal to zero, then $r_{\text {trim }}=r$. Also, it can be seen that the higher the value of the resistor in the feedback, the less significant Zload becomes.

In order to make the optimal $r_{\text {trim }}$ independent on the load, according to Eq. 28, $R_{5}$ must be equal to Eq. 30.

$$
R_{5}=r+2 R_{3}
$$

This resistor $\left(R_{5}\right)$ mimics the resistance seen from $V_{L \text { - }}$ to the ground trough $R_{1}$ and $R_{3}$ (see Fig. 9), consequently leading to $V_{L+}=V_{L-}$.

Another important characteristic of a current source is the loading capability. In bioimpedance applications, the load impedance is unknown; so on the current source must tolerate the biggest range of loads as possible. The maximum load $Z_{\text {load_max }}$ is determined by the operational amplifier output saturation $\left(V_{\text {sat }}\right)$. Eq. 31 is valid for QUAD and DIF_C topologies, while Eq. 32 is valid for DIF_M.

$$
\begin{gathered}
V_{\text {out }}=I_{\text {out }} r_{x}\left[1+\frac{z_{\text {load }}}{2 r_{x}}\left(1+\frac{r_{x}}{R_{3}}\right)\right] \leq V_{\text {sat }} \\
V_{\text {out }}=I_{\text {out }} r\left[1+\frac{z_{\text {load }}}{r}\left(\frac{r+R_{3}}{r+2 R_{3}}\right)\right] \leq V_{\text {sat }}
\end{gathered}
$$

From Figs. 7 and 8 and Eq. 31 it can easily be observed that $V_{\text {out }}$ depends on the voltage drop over the resistor $r_{x}$ plus half of the voltage drop on the load (symmetrical source). So the smaller the value of $r_{x}$ the bigger the value that $Z_{\text {load }}$ can assume. However, a key factor is the value of $R_{3}$, once it divides the value of $Z_{\text {load }}$ times $r_{x}$ increasing the value of Zload_max without changing the value of lout. For example, if $r_{x}=R_{3}$ then $Z_{\text {load }}$ is multiplied by 1 , and the advantage of using a symmetrical output current source is lost.

The equation considers an ideal operational amplifier, thus the value of Zload_max is the theoretical limit of the circuit capability for a given combination of resistors. The load range may be smaller in practical applications. Furthermore, $R_{3}$ can be chosen to improve lout, Zout and $Z_{\text {load_max }}$ without changing the value of lout.

Results and discussions

According to the equations presented in the Theory Section, the value of $R_{3}$ can lead to different output impedance, swing and common mode for the same transconductance. Also, this resistor is a positive feedback component, which can interact with the input capacitance of the amplifier and lead to instability and overshoot problems. Therefore, three values of resistors were simulated for the three proposed circuits. The values where chosen to match the available resistors with $1 \%$ tolerance.

The simulated $l_{\text {out }}$ and $Z_{\text {out }}$ can be seen in Figs. 10 and 11 , respectively. All the three sources were designed to obtain transconductance equal to $1 \mathrm{~mA} / \mathrm{V}$. The negative feedback was set to unity, so $r_{x}$ must be equal to $500 \Omega$ in the case of QUAD topology and equal to $1 \mathrm{k} \Omega$ in the case of DIF_C and DIF_M topologies. To evaluate the significance of using $r_{x}=R_{3}$ in Eq. 31 and 32, the first value of $R_{3}$ is not the same for all circuits. The second and third values are equal for a straight comparison. The input voltage was $1 V_{p}$ for all topologies and the load was $1 \mathrm{k} \Omega$. 
a)

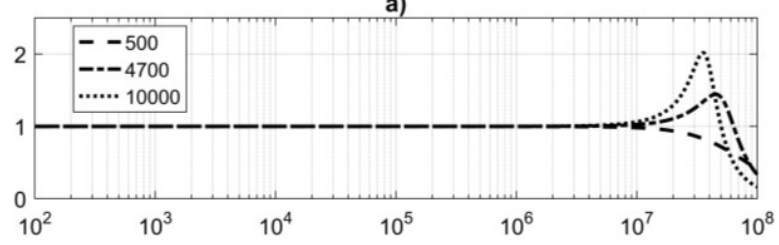

b)

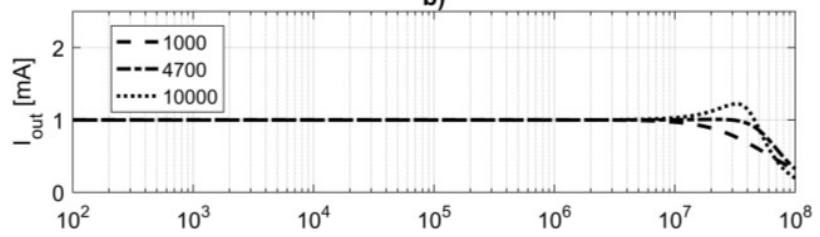

c)

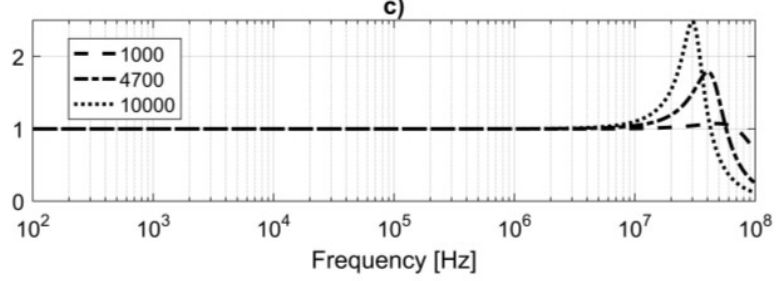

Fig.10: Simulated output current when the value of $R_{3}$ is changed. The value of $R_{3}$ in ohms is showed at the legends, the load was $1 \mathrm{k} \Omega$. a) QUAD b) DIF_C c) DIF_M.

All circuits showed a peak at high frequencies ( $>10$ $\mathrm{MHz}$ ) when a $R_{3}$ bigger than $4.7 \mathrm{k} \Omega$ is used. DIF_C was the topology with the smallest peaks, while DIF_M was the one with biggest ones. On the other hand, DIF_M showed the largest flat bandwidth.
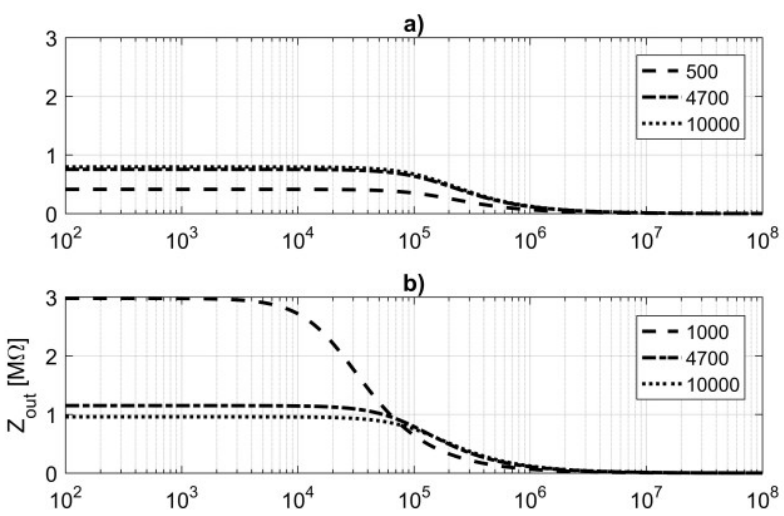

c)

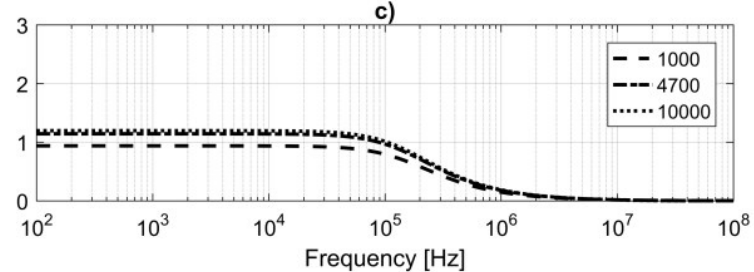

Fig.11: Simulated output impedance when the value of $R_{3}$ is changed. The value of $R_{3}$ in ohms is showed at the legends. a) QUAD b) DIF_C c) DIF_M.

QUAD and DIF_M showed an increase of output impedance when $R_{3}$ was increased, the opposite happened to DIF_C. All circuits showed values close to $1 \mathrm{M} \Omega$ for frequencies lower than $100 \mathrm{kHz}$ when $R_{3}$ is equal to $4.7 \mathrm{k} \Omega$ or $10 \mathrm{k} \Omega$. It must be said that the behavior of DIF_C in simulations using AD8132 does not match the equations. Substituting this OPAMP to an ideal one with the same open loop gain, simulation and equation results converged. By simulations, we verified that a mismatch between the two outputs of the AD8132 (not modeled by the equations) was the cause of this behavior.

In order to evaluate the influence of $R_{3}$ in Z Zload_max a simulation was performed. The load was swept from $0.1 \mathrm{k} \Omega$ to $10.1 \mathrm{k} \Omega$ in step of $500 \Omega$, Table I shows the results. The operational amplifier is powered with $\pm 5 \mathrm{~V}$, and $V_{\text {sat }}$ of the SPICE model used is $\pm 3.8 \mathrm{~V}$.

Table 1: Calculated and simulated values of $Z_{\text {load_max }}$ when varying $R_{3}$

\begin{tabular}{|l|l|c|c|c|}
\hline Circuit & $\boldsymbol{Z}_{\text {load }_{\text {max }}}[\mathrm{k} \boldsymbol{\Omega}]$ & $\boldsymbol{R}_{\mathbf{3}}=\boldsymbol{r}_{\boldsymbol{x}}$ & $\boldsymbol{R}_{\mathbf{3}}=\mathbf{4 . 7} \boldsymbol{k}$ & $\boldsymbol{R}_{\mathbf{3}}=\mathbf{1 0} \boldsymbol{k}$ \\
\hline \multirow{2}{*}{$\begin{array}{c}\text { QUAD } \\
r_{x}=500 \Omega\end{array}$} & Simulated & 3.6 & 6.1 & 6.6 \\
\cline { 2 - 5 } & Calculated & 3.3 & 6.0 & 6.3 \\
\hline \multirow{2}{*}{$\begin{array}{c}\text { DIF_C } \\
r_{x}=1000 \Omega\end{array}$} & Simulated & 3.1 & 5.1 & 5.6 \\
\cline { 2 - 5 } & Calculated & 2.8 & 4.6 & 5.1 \\
\hline DIF_M & Simulated & 4.6 & 5.6 & 5.6 \\
\cline { 2 - 5 } & Calculated & 4.2 & 5.1 & 5.4 \\
\hline
\end{tabular}

From Table 1 it can be concluded that Quad topology can drive higher loads without saturating. This can be explained by the fact that $r_{x}$ in the quad topology is half of the value in the DIF_C and DIF_M topology, thus reducing the voltage drop in this resistor. It can be seen that, in this case, the DIF_M source has less dependence on the value of $R_{3}$ then the other two sources, but its maximum load was the same as DIF_C in the simulations. It is important to notice that the deviations between calculated and simulated results are very low, meaning that the proposed mathematical equations are capable of predicting the maximum supported load.

Finally, in order to choose the value of $R_{3}$ to compare the three sources, one can choose between using the same value in all sources and using the values that result in similar and comparable behavior. In this work, the latter option was used, considering that it gives a fairer comparison in terms of common mode voltage. To put all proposed topologies in the same working conditions, a value of $R_{3}$ was defined for each one of the circuits. The parameters used to define it were the high frequency peak in the output current (considering $1 \mathrm{k} \Omega$ load), the low frequency output impedance and maximum load. Among the tested values of $R_{3}$, the ones that lead to similar response are $10 \mathrm{k} \Omega$ for DIF_C and $4.7 \mathrm{k} \Omega$ for QUAD and DIF_M.

To validate $l_{\text {out }}$ and $Z_{\text {out }}$ equations obtained for each one of the proposed circuits, they are compared to simulations, shown in Fig. 12. No variation of $R_{3}$ is done for the equations, because it is assumed that no further information would be obtained. The results can be seen in Figs. 12 and 13. The input was $1 V_{p}$ for QUAD, DIF_C and DIF_M using the inverting input, while it is used $0.333 \mathrm{~V}_{\mathrm{p}}$ for DIF_C using the non-inventing input. 

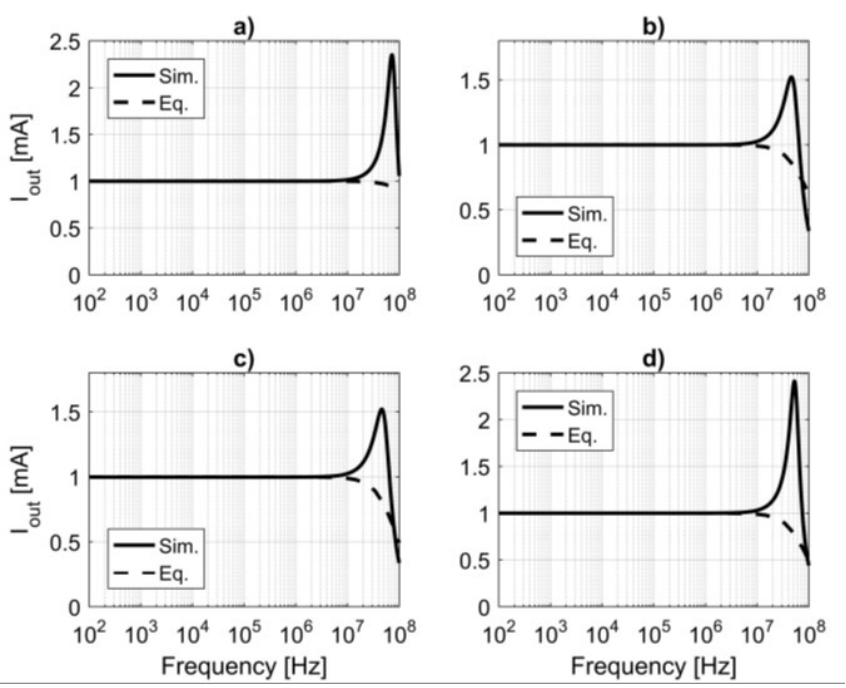

Fig.12: Simulated and calculated output current for $1 \Omega$ load. a) QUAD b) DIF_C using inverting input c) DIF_C using non-inverting input d) DIF_M.

a)

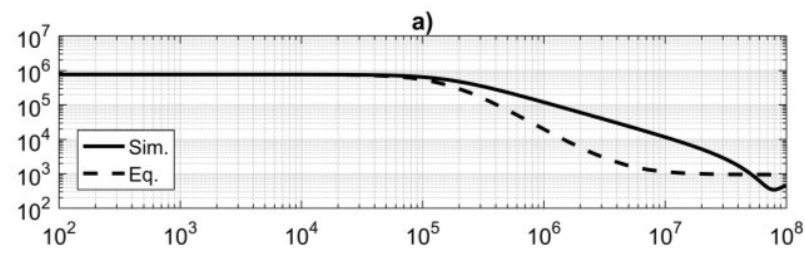

b)

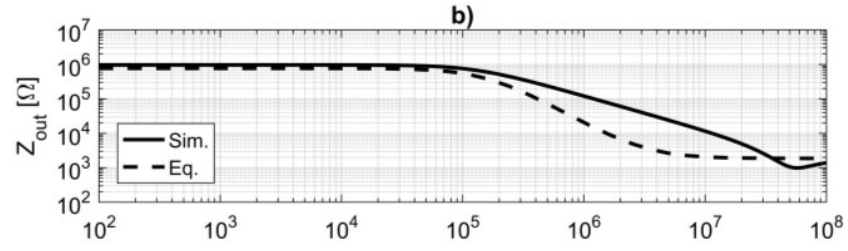

c)

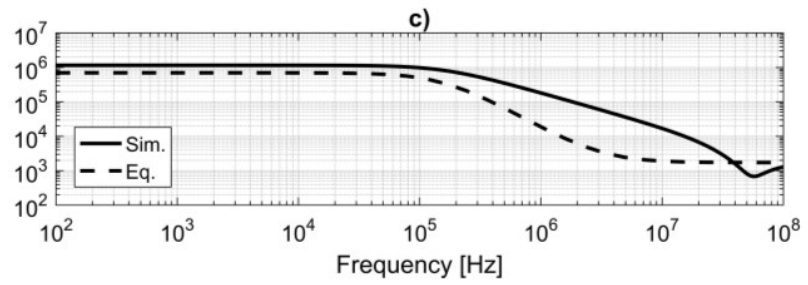

Fig.13: Simulated and calculated output impedance. a) QUAD b) DIF_Cc) DIF_M

Fig. 12 shows that the simulated and equated values for lout are very similar up to $10 \mathrm{MHz}$, where the errors begin to increase. The output impedance have similar behavior (see Fig. 13), however the frequency where the error begins to increase is approximately $100 \mathrm{kHz}$, and it can be seen that the simulated $Z_{\text {out }}$ are a little higher than the equated ones in the flat band (lower frequencies). The differences in high frequency behavior can be explained by the fact that the OPAMP input impedance is not modeled in the equations.

Once the proposed circuits are characterized, comparisons of $V_{L c m}$ between all topologies can be made. All circuits were designed to deliver an output current of 1 $m A_{p}$ for an input voltage of $1 V_{p}$. All simulated frequency analyses were done from $100 \mathrm{~Hz}$ to $100 \mathrm{MHz}$, while in the time analysis it was set to $1 \mathrm{MHz}$. The load was fixed at 1 $k \Omega$, except for load dependence experiments where four loads were used: $0.1 \mathrm{k} \Omega, 1.6 \mathrm{k} \Omega, 3.1 \mathrm{k} \Omega$ and $4.6 \mathrm{k} \Omega$.

The results are shown in graphs containing the circuits of the same group (non-differential, mirrored, proposed) and then they are discussed against each other. The frequency response of $V_{L c m}$ for the circuits in the nondifferential group is shown in Fig. 14. The resistor $r_{\text {trim }}$ is equal to $r(1 \mathrm{k} \Omega)$ for EHCS-DIF and BRIDGE-1.

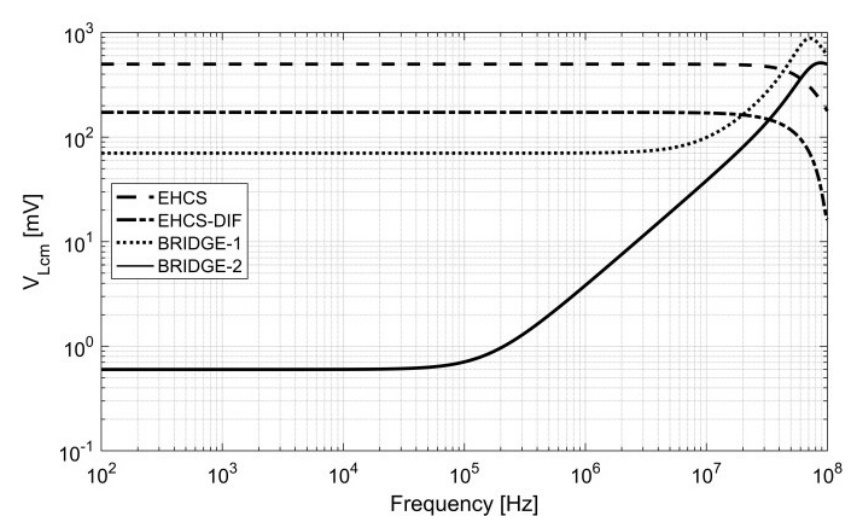

Fig.14: Simulated common mode load voltage $\left(V_{L \mathrm{~cm}}\right)$ of the nondifferential EHCS for a load of $1 \mathrm{k} \Omega$.

In Fig. 14, the EHCS had the highest $V_{L c m}, 0.5 \mathrm{~V}$, which is half of the load voltage as expected. EHCS-DIF and BRIDGE1 showed responses of about $200 \mathrm{mV}$ and $70 \mathrm{mV}$, respectively. BRIDGE-2 had an increase on common mode voltage after $100 \mathrm{kHz}$, but remained the smallest $V_{L c m}$ until $30 \mathrm{MHz}$. The waveform of $V_{L c m}$ for the non-differential group is shown in Fig. 15, including the DC component.

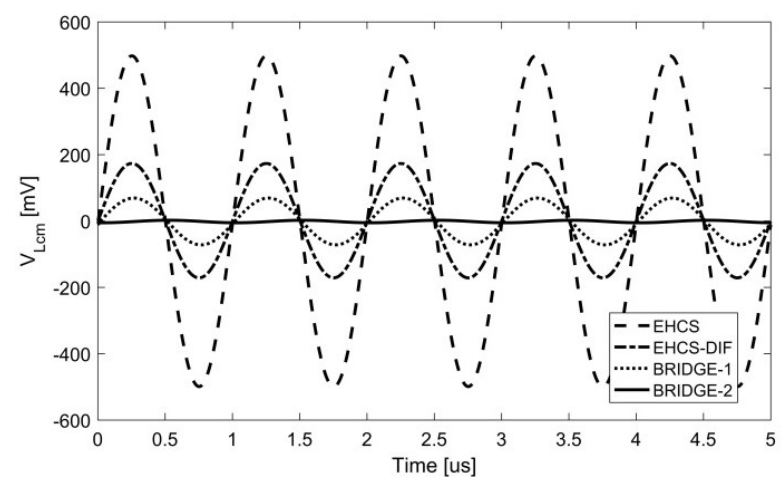

Fig.15: Simulated common mode load voltage $\left(V_{\mathrm{Lcm}}\right)$ of the nondifferential EHCS in time domain at $1 \mathrm{MHz}$ for a load of $1 \mathrm{k} \Omega$.

It can be seen in Fig. 15 that the $A C$ values match the ones shown in Fig. 14 at $1 \mathrm{MHz}$, and that the DC component of $V_{L c m}$ is almost null compared to the AC component. As the output current is the same for all the circuits, BRIDGE-2 presented the best common mode rejection of the nondifferential group. However, note that the reduction in common mode voltage is at cost of complexity, as the number of active components increases (BRIDGE-1 and BRIDGE-2 have two and three OPAMPS, respectively). 
Although $r_{\text {trim }}$ can be changed to mimic the voltage drop at $r$, this voltage drop is not constant for different loads. A load variation was simulated to study how it affects $V_{L c m}$.

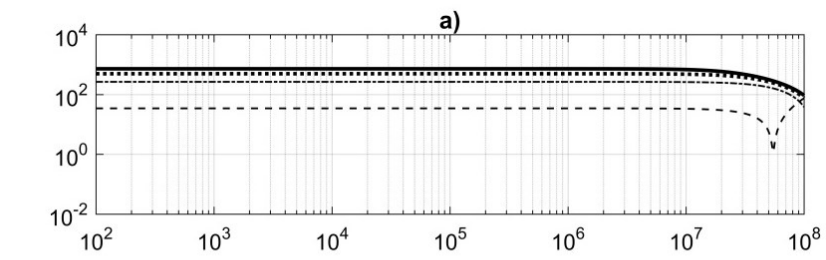

b)

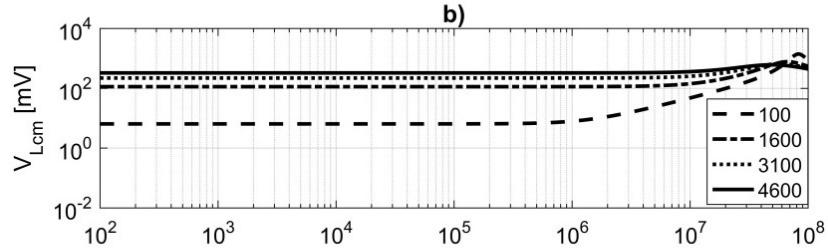

c)

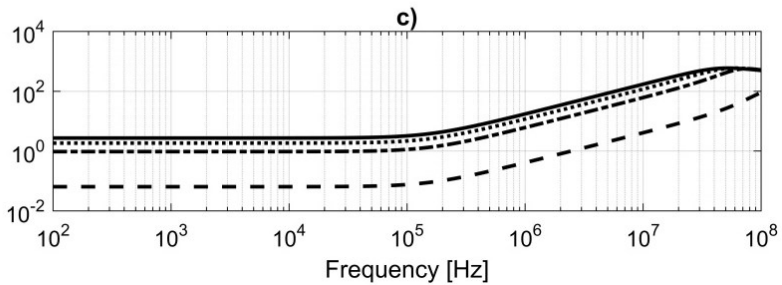

Fig.16: Simulated common mode load voltage $\left(V_{L c m}\right)$ of the nondifferential EHCS for four different loads. The values shown in the legend are the value of the loads in $\Omega$. a) EHCS-DIF $b$ ) BRIDGE-1 c) BRIDGE-2.

In Fig. 16, it can be seen that all circuits showed a large variation of $V_{\mathrm{Lcm}}$ for the step of $0.1 \mathrm{k} \Omega$ to $1.6 \mathrm{k} \Omega$ and small variations for the others load steps. BRIDGE-2 showed values smaller than $10 \mathrm{mV}$ for all loads until $100 \mathrm{kHz}$, but for higher frequencies it raises to close to $1 \mathrm{~V}$. With exception of $100 \Omega$, EHCS-DIF and BRIDGE- 1 show almost constant values, around $1 \mathrm{~V}$ and $0.1 \mathrm{~V}$, respectively.

In Fig. 17, the AC common mode analysis is shown for the other two groups (Mirrored and Proposed group). MEHCS-DIF was excluded because the result was exactly the same as for MEHCS.

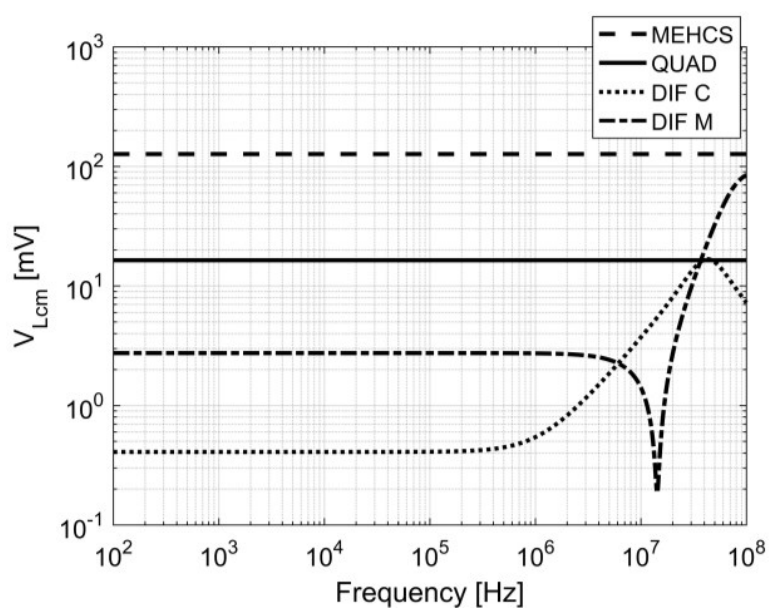

Fig.17: Simulated AC common mode load voltage $\left(V_{L c m}\right)$ of the mirrored group and proposed group. MEHCS-DIF and MEHCS have the same curve.
In Fig. 17, it is shown that the proposed DIF_C topology has the lowest $V_{L c m}$; at the flat zone it is equal to $0.4 \mathrm{mV}$, rising to around $20 \mathrm{mV}$ in high frequencies. QUAD and MEHCS showed constant $V_{\text {Lcm }}$ of 16.5 and $120 \mathrm{mV}$ respectively, along all frequencies. DIF_M showed slightly higher values than DIF_C, about $3 \mathrm{mV}$. In Fig. 18, the time analysis is shown for these two groups.
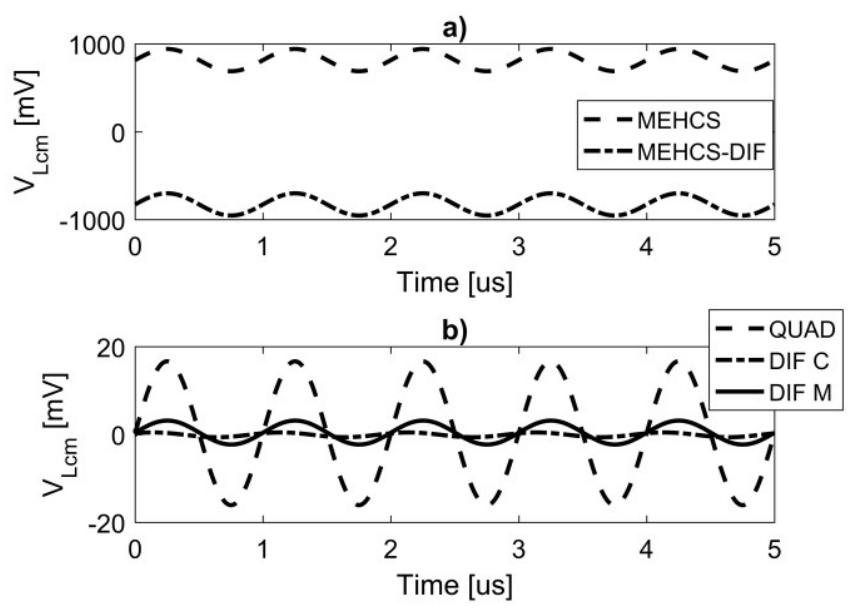

Fig.18: Simulated common mode load voltage $\left(V_{L \mathrm{~cm}}\right)$ of the mirrored group and proposed group at time domain in $1 \mathrm{MHz}$.

In Fig. 18 b) it can be seen that all proposed topologies do not show significant DC component, in contrast with the mirrored circuits, Fig. 18 a), with DC value around \pm 800 $\mathrm{mV}$. The DC component of the MEHCS source is a consequence of the offset signal of the two amplifiers used in the configuration, which creates a common mode output current that flows through the output impedance of each EHCS section. The AC amplitudes agree with the values seen in Fig. 17 at $1 \mathrm{MHz}$.

To analyze the effect of a resistor mismatch, Fig. 19 shows the common mode frequency response of both mirrored and proposed group when a $\pm 1 \%$ error is applied to the resistors $r$ and $r_{x}$.

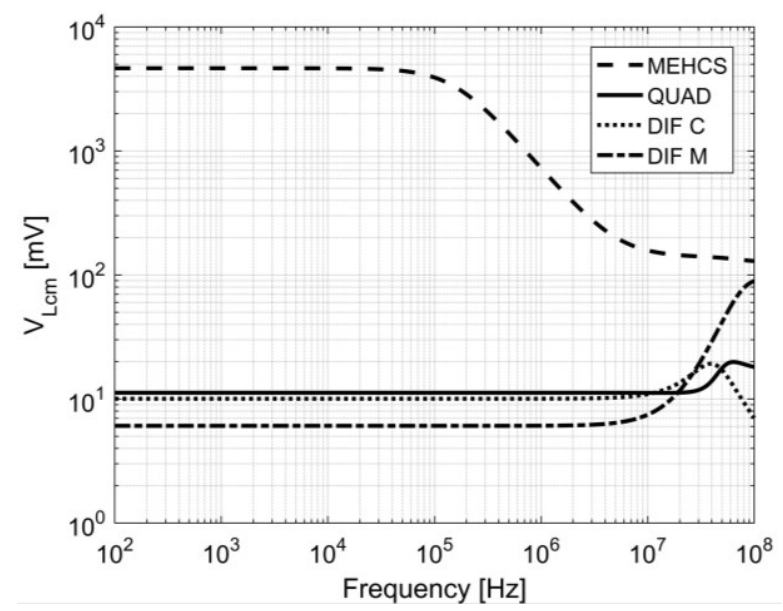

Fig.19: Simulated AC common mode load voltage $\left(V_{L c m}\right)$ of the mirrored group and proposed group, with unmatched control resistors ( $r$ and $r_{x}$ ). 
In figure 19, DIF_M shows the smallest $V_{L c m}$ in most frequencies, from 6 to $90 \mathrm{mV}$, while DIF_C varies from 10 to $20 \mathrm{mV}$. Quad is almost constant at $11 \mathrm{mV}$, while MEHCS falls from 4600 to $130 \mathrm{mV}$. This shows that the MEHCS circuit is very sensitive to resistor mismatch in terms of common mode signal, whereas the DIF_M source shows a robust behavior. Furthermore, the common mode voltage of the mismatched MEHCS was almost one order of magnitude larger than the other circuits, which shows that this source is much less effective in common mode reduction in practical situations. Fig. 20 presents the time analysis for both groups when $r$ and $r_{x}$ are unmatched with $\pm 1 \%$.
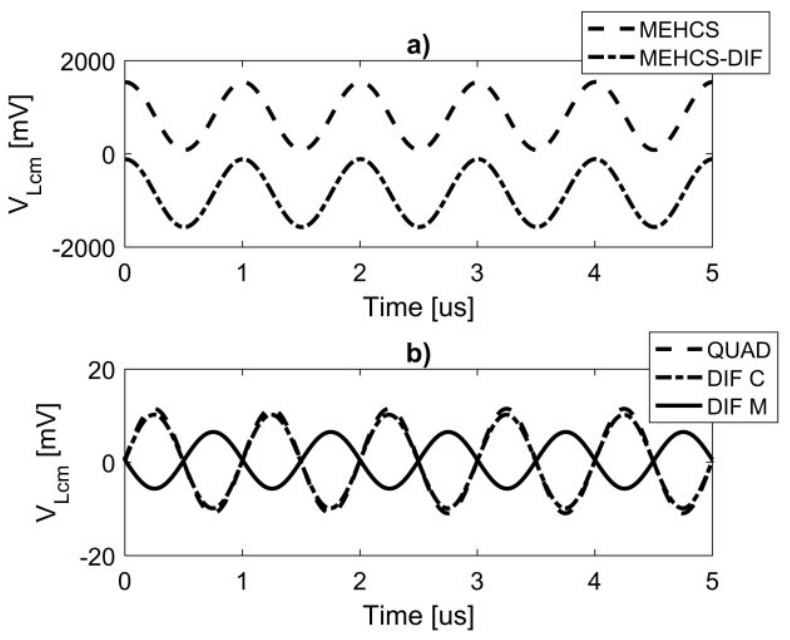

Fig.20: Simulated common mode load voltage $\left(V_{L c m}\right)$ of the mirrored group and proposed group at time domain in $1 \mathrm{MHz}$ with unmatched control resistors ( $r$ and $r_{x}$ ).

It can be seen that even with unmatched sides, the proposed topologies has not shown any significant DC component, while for MEHCS and MEHCS-DIF it is maintained at $\pm 800 \mathrm{mV}$. This DC signal is usually not desirable in bioimpedance applications, as it decreases output swing and requires the use of series blocking capacitors to isolate the load.

Fig. 21 shows the common mode response using four different loads $(0.1 \mathrm{k} \Omega, 1.6 \mathrm{k} \Omega, 3.2 \mathrm{k} \Omega$ and $4.6 \mathrm{k} \Omega$ ) for DIF_C, QUAD and DIF_M.

In Fig. 21 it can be seen that both QUAD and DIF_C showed $V_{L c m}$ around $10 \mathrm{mV}$ for all four loads. On the other hand DIF_M showed higher dependence on the load; yet, all values of $V_{L c m}$ are smaller than the ones obtained with QUAD and DIF_C. This response, in contrast with the ones presented in Fig. 16, shows that the circuits of the proposed group have a common mode response much less dependent on the load than the ones from the nondifferential group, suggesting that the proposed group is more suitable to applications that requires low common mode voltage with large load ranges.

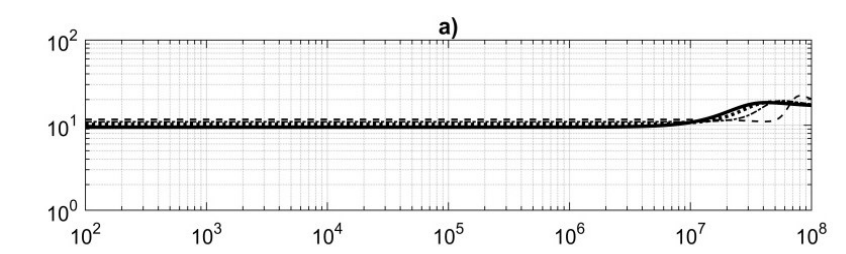

b)
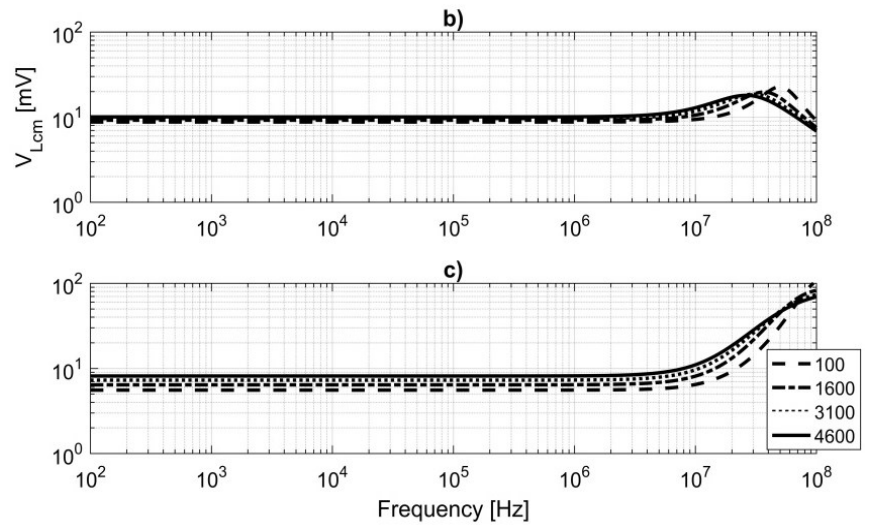

Fig.21: Simulated common mode load voltage $\left(V_{L c m}\right)$ of the proposed group, with unmatched $( \pm 1 \%$ ) control resistors ( $r$ and $r_{x}$ ), for four different loads. The label shows the values of the loads in $\Omega$. a) QUAD b) DIF_C c) DIF_M.

In order to analyze the behavior of the common mode voltage when submitted to random variations in the resistors values (due to fabrication tolerance), a Monte Carlo simulation was performed (1000 rounds) in the mirrored and proposed groups, considering all the resistors in the circuits with $1 \%$ tolerance (Gaussian distribution of resistor values). The results are shown on the histograms of Fig. 22. The common mode values presented in this case were the maximum absolute value in the range of $100 \mathrm{~Hz}$ to $1 \mathrm{MHz}$. It can be seen that the mean value of the proposed group common-mode voltage is similar to the ones found in Fig. 19, and even the worst case is kept below $30 \mathrm{mV}$ using relatively common resistors. On the other hand, the mirrored circuit showed a significantly larger variation, with $15 \%$ of the values outside the linear range of the operational amplifier, which is $5 \mathrm{~V}$. The histograms of Fig. 22 show values above $5 \mathrm{~V}$ because the $A C$ simulation of PSPICE does not consider saturation limits of the amplifier. This means that the mirrored group can easily saturate with random resistor variations caused by tolerance.

Once the simulations showed that the proposed circuits are able to reduce the common mode voltage and enhance output swing, experimental data with practical circuits was collected. The proposed group circuits were implemented with resistor values to match the simulations ( $1 \%$ tolerance), using AD8132 differential amplifiers and doublelayer boards to accommodate a ground plane. The resistors used as loads were measured previously to the tests, resulting in the values $100,995,2492$ and $4692 \Omega$. Due to parasitic components and limitation of the instruments, the experimental data was limited to $1 \mathrm{MHz}$. 

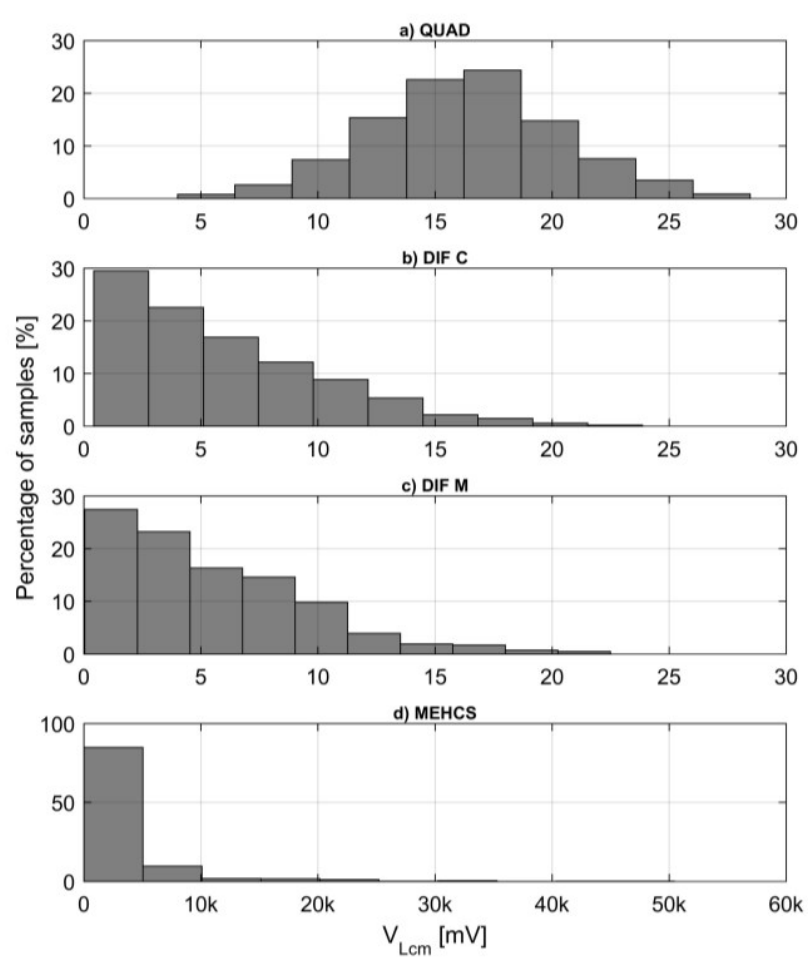

Fig. 22: Common mode voltage distribution using Monte Carlo simulation considering resistors with $1 \%$ tolerance.
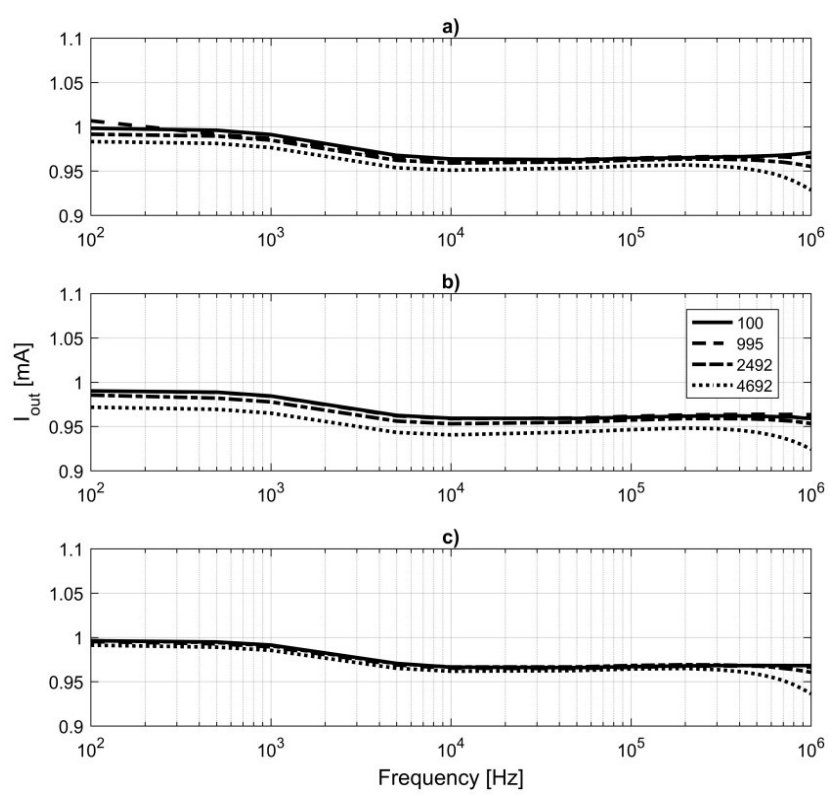

Fig.23: Measured output current for different loads (100, 995, 2492 and $4692 \Omega$ ). a) QUAD b) DIF_C c) DIF_M.

In Fig. 23, the output current of the proposed circuits for four different loads is shown.

The DIF_M showed the smallest dependence on the load, while DIF_C showed the largest. All topologies showed a decrease in the output current at $1 \mathrm{MHz}$ for a load of 4.7 $k \Omega$, evidencing the influence of a high frequency pole dependent on the load value. In Fig. 24, the output current phase can be seen.

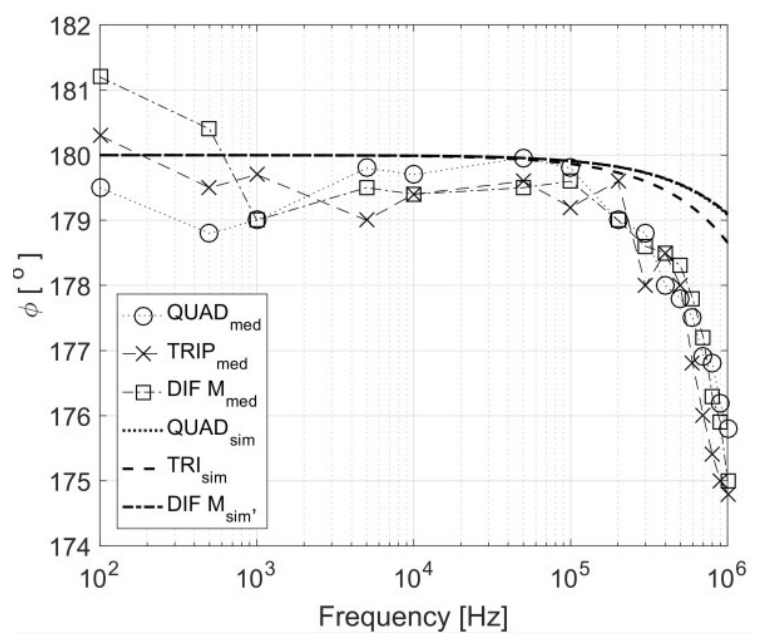

Fig.24: Measured and simulated output current phase for a $1 \mathrm{k} \Omega$ load. a) QUAD b) DIF_C c) DIF_M.

The increasing deviation in high frequencies suggests that the pole of the practical circuit is slightly lower that the simulated, which was expected due to parasitic components. The simulated output current phase of QUAD and DIF_M are overlapped, while DIF_C showed a slightly bigger decrease close to $1 \mathrm{MHz}$.

From the magnitude variation of output current using different loads, the output impedance can be obtained, and the results are shown in Fig. 25. They are obtained by an equation that considers that the output impedance is in parallel with the load, the load voltage and the load impedance are necessary.

$$
Z_{\text {out }}=\frac{V_{L_{2}}-V_{L_{1}}}{V_{L_{1}} / R_{L_{1}}-{ }^{V_{L_{2}} / R_{L_{2}}}}
$$

Where $V_{L 2}$ is the measured load voltage for the biggest load $\left(R_{L 2}=4.7 \mathrm{k} \Omega\right)$ and $V_{L 1}$ is the load voltage for the smallest load $\left(R_{L 1}=100 \Omega\right)$.

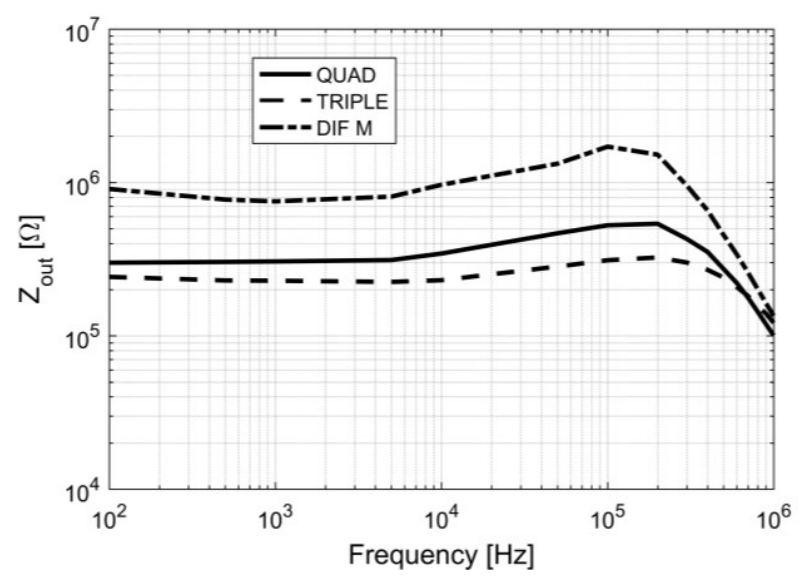

Fig.25: Measured output impedance. a) QUAD b) DIF_C c) DIF_M

The DIF_M topology showed the highest output impedance, near $1 \mathrm{M} \Omega$ from $100 \mathrm{~Hz}$ to $100 \mathrm{kHz}$, while DIF_C 
and QUAD showed values considerably smaller, $300 \mathrm{k} \Omega$ and $400 \mathrm{k} \Omega$ in the same frequency region.

The output common mode voltage was obtained using Eq. 4, and is shown in Fig. 26. The measurements were divided by the input voltage, to compensate for small variations of the voltage generator in the frequency range.
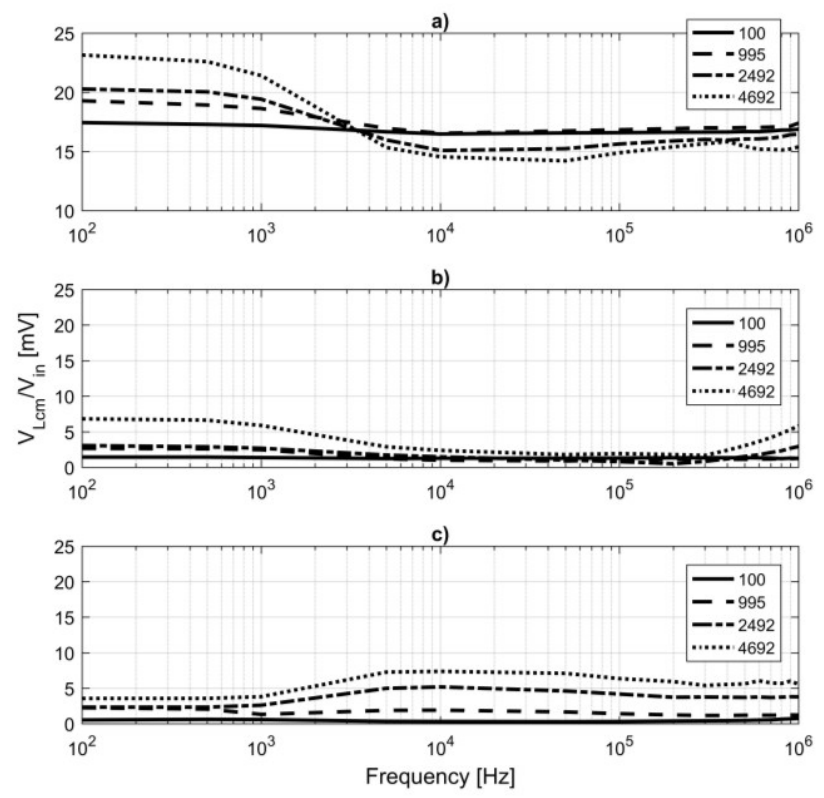

Fig.26: Measured load common mode voltage. a) QUAD b) DIF_C c) DIF_M

The common mode voltages of DIF_M and DIF_C are both smaller than $8 \mathrm{mV}$, while QUAD showed higher maximum value, $23 \mathrm{mV}$ (in $100 \mathrm{~Hz}$ ). Although the magnitude values are similar to simulations (see Fig. 17 and 19), the behavior of the curves are different from the expected. One possible explanation is the limited precision of the instrumentation, as the common mode value are much lower than the differential signal measured. However, if this signal is treated as undesirable noise in an application, only the maximum range of values that the common mode voltage can assume in the frequency band is important, and not its dependence on the frequency.

A complementary characteristic that can be obtained is the CMRR. This parameter indicates the relation between the differential load voltage, the signal of interest in bioimpedance applications, and the output common mode voltage, undesirable signal that can generate artifacts and noise in a measurement. These results can be seen in Fig. 27.

From the results, one can conclude that the CMRR is highly dependent on the load impedance in all circuits tested. This behavior was expected from the previous results, as the load differential voltage increases linearly with the load, while the load common mode voltage dependence on the load is more complex (see Fig. 26). QUAD showed the smallest CMRR while DIF_C showed the highest.
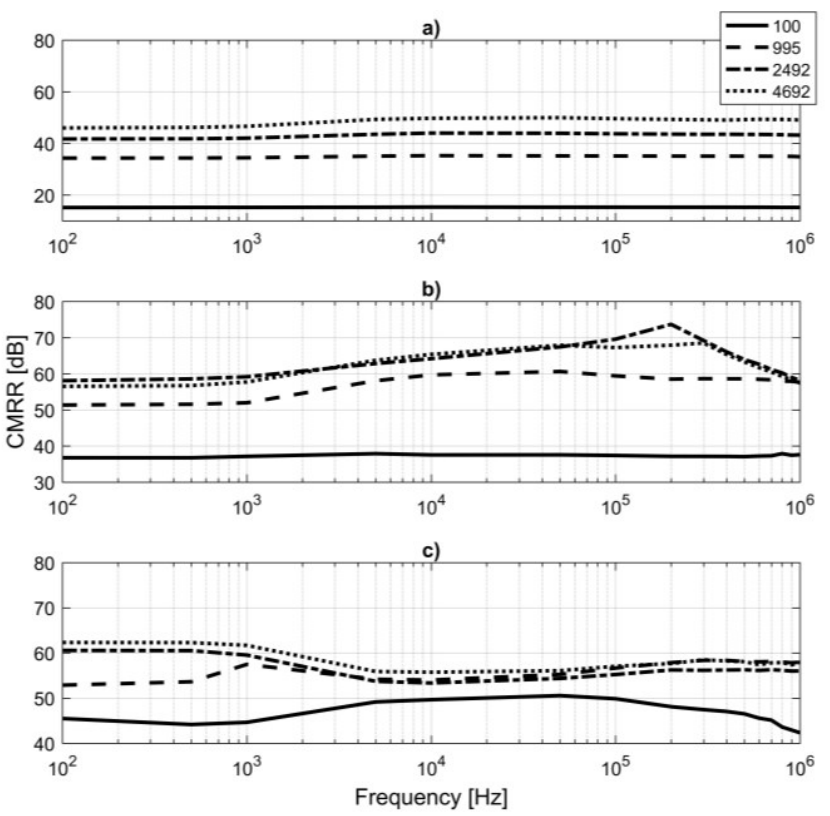

Fig.27: Measured common mode rejection ratio (CMRR). a) QUAD b) DIF_C c) DIF_M

It is important to notice that the DIF_M circuit showed the highest CMRR using the lowest load, which is desirable considering that common mode problems are significant with small differential signals.

Bioimpedances are usually in the range of 10 to $1000 \Omega$, depending on the application, so using DIF_C or DIF_M topologies could enhance the CMRR up to $60 \mathrm{~dB}$ when compared to EHCS (CMRR $\approx 6 \mathrm{~dB}$ )

The other property of the Howland current source that is being investigated is the output swing; the measured ones are shown in Table 2.

Table 2: Measured values of $Z_{\text {load_max }}$

\begin{tabular}{ccc}
\hline QUAD $[\mathrm{k} \Omega]$ & DIF_C $[\mathrm{k} \Omega]$ & $\mathrm{DIF}-\mathrm{M}[\mathrm{k} \Omega]$ \\
6.31 & 5.33 & 5.16 \\
\hline
\end{tabular}

QUAD showed the highest Zload_max, mainly due to $r_{x}$ being half of the ones used in the other topologies, while DIF_C showed a small enhancement in comparison to DIF_M.

Furthermore, the topologies DIF_C and QUAD have both outputs working in high impedance, while DIF_M have one output at high impedance and the other one in low impedance. It could not be found in the literature whether this characteristic has any practical meaning in bioimpedance measurements.

\section{Conclusion}

In this work, it has been found that from the Howland current sources topologies found in literature, the one that showed best common mode rejection was the BRIDGE-2, but this circuit requires three operational amplifiers. Three new circuits are proposed, all able to reach BRIDGE-2 common mode rejection using only one operational 
amplifier. All three proposed circuits showed close results, the intended application will determine which one is better.

\section{Acknowledgment}

We would like to thanks UDESC for the scholarship and our advisor Pedro Bertemes-Filho for the position at the group and laboratory.

\section{Conflict of interest}

Authors state no conflict of interest.

\section{References}

1. Boone KG., Holder DS. Current approaches to analogue instrumentation design in electrical impedance tomography. Physiological measurement, 1996, 17(4): 229. https://doi.org/10.1088/0967-3334/17/4/001.

2. Aberg $\mathrm{P}$, et al. Skin cancer identification using multifrequency electrical impedance-a potential screening tool. IEEE transactions on biomedical engineering, 2004, 51(12): 20972102. https://doi.org/10.1109/TBME.2004.836523

3. Yang $\mathrm{Y}, \mathrm{Wang} \mathrm{J}$. A design of bioimpedance spectrometer for early detection of pressure ulcer. In: Engineering in Medicine and Biology Society, 2005. IEEE-EMBS 2005. 27th Annual International Conference of the. IEEE, 2006. p. 6602-6604.

4. Seoane F; Bragós R; Lindecrantz K. Current source for multifrequency broadband electrical bioimpedance spectroscopy systems. A novel approach. In: Engineering in Medicine and Biology Society, 2006. EMBS'06. 28th Annual International Conference of the IEEE. IEEE, 2006. p. 51215125. https://doi.org/10.1109/IEMBS.2006.259566.

5. Mohamadou $Y$, et al. Performance evaluation of wideband bio-impedance spectroscopy using constant voltage source and constant current source. Measurement Science and Technology, 2012, 23.10: 105703.

6. Sansen W, Geeraerts B, Van Petegem W, Dehaene W, Steyaert M. Voltage versus current driven high frequency EIT systems. In: Biomedical Engineering Days, 1992. Proceedings of the 1992 International. IEEE, 1992. p. 102-106. https://doi.org/10.1109/IBED.1992.247078

7. Qureshi TR, Chatwin C \& Wang W. (2013). Bio-impedance excitation system: A comparison of voltage source and current source designs. APCBEE Procedia, 7, 42-47. https://doi.org/10.1016/j.apcbee.2013.08.010

8. Mohamadou Y, Oh TI, Wi H, Sohal H, Farooq A, Woo EJ \& McEwan AL (2012). Performance evaluation of wideband bioimpedance spectroscopy using constant voltage source and constant current source. Measurement Science and Technology, 23(10), 105703.

https://doi.org/10.1088/0957-0233/23/10/105703

9. Hong H, Demosthenous A, Triantis IF, Langlois P, Bayford R. A high output impedance $C M O S$ current driver for bioimpedance measurements. In: Biomedical Circuits and Systems Conference (BioCAS), 2010 IEEE. IEEE, 2010. p. 230233. https://doi.org/10.1109/BIOCAS.2010.5709613

10. Tucker AS, Fox RM, Sadleir RJ. Biocompatible, high precision, wideband, improved Howland current source with lead-lag compensation. IEEE Transactions on Biomedical Circuits and
Systems, 2013, 7.1: 63-70.

https://doi.org/10.1109/TBCAS.2012.2199114

11. Bertemes-Filho P, Felipe A, Vincence VC. High accurate Howland current source: Output constraints analysis. Circuits and Systems, 2013, 4.07: 451

12. Constantin AV, Gheorghe GI. Simulations of basics topologies and method for practical determination of the output impedance for Howland current sources used for chemical microsensors and biomedical application. In: International Semiconductor Conference (CAS), 2016. IEEE, 2016. p. 183186. https://doi.org/10.1109/SMICND.2016.7783080

13. Morcelles KF, Sirtoli VG, Bertemes-Filho P, Vincence VC. Howland current source for high impedance load applications. Review of Scientific Instruments, 2017, 88.11: 114705. https://doi.org/10.1063/1.5005330

14. Bertemes-Filho P, Lima RG, Tanaka H. A Current Source using a Negative Impedance Converter (NIC) for Electrical Impedance Tomography (EIT). In: Proceedings of the 17th International Congress on Mechanical Engineering. São Paulo. 2003. p. 83-7.

15. Qureshi TR, Chatwin CR, Huber N, Zarafshani A, Tunstall B, Wang W. Comparison of Howland and General Impedance Converter (GIC) circuit based current sources for bioimpedance measurements. In: Journal of Physics: Conference Series. IOP Publishing, 2010. p. 012167. https://doi.org/10.1088/1742-6596/224/1/012167

16. Pliquett $U$, Schönfeldt $M$, Barthel $A$, Frense $D$, Nacke $T$. Offset-free bidirectional current source for impedance measurement. In: Journal of Physics: Conference Series. IOP Publishing, 2010. p. 012009. https://doi.org/10.1088/1742-6596/224/1/012009

17. Liu J, Qiao X, Wang M, Zhang W, Li G, Lin L. The differential Howland current source with high signal to noise ratio for bioimpedance measurement system. Review of Scientific Instruments, 2014, 85.5: 055111.

18. Pliquett $U$, Schönfeldt $M$, Barthel $A$, Frense $D$, Nacke $T$ \& Beckmann D. Front end with offset-free symmetrical current source optimized for time domain impedance spectroscopy. Physiological measurement, 32(7), 927. https://doi.org/10.1088/0967-3334/32/7/S15

19. Zhang F, Teng Z, Zhong H, Yang Y, Li J \& Sang J (2018). Wideband mirrored current source design based on differential difference amplifier for electrical bioimpedance spectroscopy. Biomedical Physics \& Engineering Express, 4(2), 025032. https://doi.org/10.1088/2057-1976/aaa9cd

20. Lamlih A, Freitas P, David-Grignot S, et al. (2018, May). Wideband Fully Differential Current Driver with Optimized Output Impedance for Bioimpedance Measurements. In Circuits and Systems (ISCAS), 2018 IEEE International Symposium on (pp. 1-5). IEEE. https://doi.org/10.1109/ISCAS.2018.8351464

21. Li X, Dong F \& Fu Y. (2012, May). Analysis of constant-current characteristics for current sources. In Control and Decision Conference (CCDC), 2012 24th Chinese (pp. 2607-2612). IEEE. https://doi.org/10.1109/CCDC.2012.6244414

22. Rafiei-Naeini M \& McCann H (2008). Low-noise current excitation sub-system for medical EIT. Physiological measurement, 29(6), S173. https://doi.org/10.1088/0967-3334/29/6/S15 